ISSN: $1130-3743$

DOI: http://dx.doi.org/10.14201/teoredu2014261205228

\title{
COMPETENCIAS BÁSICAS Y CURRÍCULUM. EL CASO DE CATALUÑA
}

\author{
Basic competencies and curricula. The case of Catalonia
}

\section{Les compétences basiques et les programmes de l'école. Le cas de la Catalogne}

Jaume SARRAMONA I LÓPEZ

Universitat Autònoma de Barcelona. Departament de Pedagogia Sistemàtica i

Social. Edifici G6. 08193 Bellaterra (Cerdanyola del Vallès).

jaumesarralo@gmail.com.

Fecha de recepción: marzo de 2014

Fecha de aceptación: junio de 2014

Biblid [(1130-3743) 26, 2-2014, 205-228]

RESUMEN

El artículo plantea las cuestiones que más habitualmente son objeto de polémica al tratar el tema de las competencias, aunque ya se trata de un concepto ampliamente adoptado en los sistemas educativos. También se propone una clarificación de los distintos calificativos que se otorgan a las competencias, en función de su ámbito de aplicación, así como el análisis de cuál es su aportación real al avance pedagógico.

La segunda parte del artículo presenta el caso de Cataluña como una experiencia de integración de las competencias básicas en el currículum, con la novedad de su clara formulación vinculadas a las diversas áreas o materias, así como su propuesta de gradación en tres niveles de consecución.

Palabras clave: currículum; competencias; competencias básicas; evaluación de competencias; indicadores de logro; gradación de las competencias. 
SUMMARY

The article raises issues that are usually considered controversial to address the issue of competencies, although it is a widely adopted concept in educational systems. Clarification of the various denominations that are granted the powers, depending on its scope and analysis of what their real contribution to educational progress is also proposed.

The second part of the paper presents the case of Catalonia as an experience of integration of basic competencies in the currículum, with the novelty of his clear formulation related to the various areas or subjects, as well as its proposed gradation into three achievement levels.

Key words: currículum; competencies; basic competencies; competency assessment; performance indicators; gradation of competencies.

SOMMAIRE

L'article soulève des questions qui sont habituellement considérés controversées d'aborder la question des compétences, bien qu'il soit largement adopté dans le concept des systèmes éducatifs. Clarification des différentes confessions qui sont accordés des pouvoirs, en fonction de son champ d'application et l'analyse de ce que leur contribution réelle à l'avancement de l'éducation est également proposé.

La deuxième partie de l'article présente le cas de la Catalogne comme une expérience de l'intégration des compétences de base dans le programme, avec la nouveauté de ses termes clairs liés aux divers domaines ou des sujets, ainsi que ses gradation proposée trois niveaux de rendement.

Mots clés: programmes de l'école; les compétences; les compétences basiques; évaluation des compétences; indicateurs de performance; gradation des compétences.

1. UNAS CONSIDERACIONES PREVIAS SOBRE LAS COMPETENCIAS, QUE AÚN RESULTAN NECESARIAS

La cantidad de publicaciones sobre competencias empieza a ser realmente abrumadora, con el inconveniente del ya conocido principio de que mucha información no siempre aclara el conocimiento sino que lo puede sumergir en un mar de ruido desconcertante. Esto no tendría mayor trascendencia si se tratara de una cuestión meramente conceptual, pero resulta que las competencias han penetrado como objetivos a alcanzar en el sistema educativo en todos sus niveles, desde la enseñanza primaria hasta los doctorados universitarios, y hay pensar que para quedarse un cierto tiempo; consecuentemente, deberían tener implicaciones en la práctica totalidad de la docencia. Se trata de lo que el comparativista catalán Pere Rosselló llamaría una "corriente educativa", de una indudable fuerza. La conclusión es obvia: o clarificamos bien qué queremos decir al hablar de competencias en la 
enseñanza y las hacemos comprensibles y relativamente fáciles de ser llevadas a la práctica, o se convertirán en una simple jerga para ser depositada sobre publicaciones académicas, documentos oficiales y propuestas curriculares, sin trascendencia real sobre la práctica pedagógica.

El término "competencia" no tiene mucha tradición en el sistema educativo, a excepción de la formación profesional, donde hace muchos años que ya está instalado. De hecho, nos viene del mundo del trabajo, del mundo económico, del mundo empresarial, donde la competitividad resulta necesaria para la supervivencia y se asocia a la calidad. En el lenguaje cotidiano se califica de "competente" aquel profesional que resuelve bien los problemas que son propios de su ámbito de actuación. Este origen y vinculación con el mundo económico ha sido una fuente de crítica a la hora de aplicar las competencias a la educación, bajo la acusación de querer importar criterios economicistas y empresariales, haciendo perder así a la escuela su naturaleza formativa. Se trata de una crítica ideológica que se desvanece cuando el concepto de competencias se analiza en perspectiva estrictamente pedagógica y se une a determinados calificativos, como el de "básicas", en los niveles de la enseñanza obligatoria, o al de "profesionales", en el caso de la enseñanza profesionalizadora. No seguiremos por este camino; los riesgos que señalan los detractores son claramente inferiores a las ventajas que el concepto nos aporta y más cuando no se conciben como el único tipo de aprendizajes a lograr en el sistema educativo. Con todo, convendrá hacer algunas consideraciones al respecto.

Unos términos próximos al de competencia son competir y competitividad. Competir significa entrar en comparación con alguien, lo que lleva implícito el deseo de superar o vencer. La competición es inherente al deporte, por ejemplo, pero la tenemos instaurada en la inmensa mayoría de las actividades de nuestra sociedad, empezando por la economía y siguiendo por los terrenos científicos, académicos, políticos, ideológicos, etc. Se sabe que una empresa u organismo no competitivos están condenados al fracaso; guste o no, hay que competir con la "competencia" (la palabra ya es suficientemente explícita) para sobrevivir en un mundo globalizado, de libre circulación de conocimientos, personas y bienes. Los países enteros se esfuerzan en ser competitivos para avanzar en el camino de las relaciones internacionales, y en el mismo mundo académico los centros educativos de todos los niveles compiten para conseguir alumnos y recursos. La propia declaración de Bolonia (1999) sobre los sistemas universitarios afirmaba que "la adopción de un sistema de titulación fácilmente legible y homologable, incluso mediante la implementación del Diploma Supplement, para favorecer la empleabilidad de los ciudadanos europeos y la competitividad internacional del sistema europeo de la enseñanza superior".

Este concepto de competitividad afecta al sistema educativo?; debe preparar la escuela para competir? Inicialmente podría parecer lógico afirmar que la educación escolar no puede cerrar los ojos a la realidad social de la competitividad, porque si lo hace no cumple con su objetivo general de preparar para la vida cotidiana en 
todas sus facetas. Pero es igualmente cierto que la escuela no es una institución puesta al servicio de los intereses del mercado, ni su funcionamiento debe responder a los mismos criterios que los organismos sociales que están en situación de competencia en este mercado.

Tradicionalmente la escuela ha considerado la competencia entre sujetos como una dimensión de la necesaria emulación para superarse. La competición individual o en equipo se ha aplicado en la llamada "escuela tradicional" como una forma habitual de motivación, no tanto fundamentada en la existencia de una sociedad competitiva como por la concepción de la misma competencia como algo positivo para al desarrollo personal. Pero actualmente, por suerte, podemos afirmar que en la vida escolar ha calado ampliamente la preponderancia del principio de colaboración, de ayuda a los demás, de modo que se plantea el trabajo en equipo como materialización de unas metas educativas y no como una forma de organización para competir. Aunque la escuela es una organización social que se ve afectada por criterios dominantes del contexto, tiene una misión específica muy diferente a la estrictamente económica y más bien establecerá las bases para desarrollar una actitud crítica respecto a la competitividad salvaje y sus equivalentes de "todo para la venta" (no muy diferente, por ejemplo, el conocido "todo por la audiencia", que practican muchos medios de comunicación). En síntesis, la pedagogía actual considera que la escuela no tiene que preparar para competir en la vida social sino que debe fomentar los más nobles valores sociales de la ayuda y la colaboración; en todo caso ya será el mundo laboral el que preparará a los sujetos que entren en él para la supervivencia y el progreso, de acuerdo con los parámetros que allí rigen.

¿Esto quiere decir que en la escuela no se puede utilizar la emulación para fomentar el aprendizaje y la mejora personal? Por supuesto que se puede utilizar. Pero la emulación o espíritu de superación debe centrarse en uno mismo, en un legítimo deseo de mejora constante, y no necesariamente en la comparación con los demás. Competir con uno mismo constituye un sano principio de estímulo personal, básico para seguir aprendiendo y mejorando, sin que se llegue a perder el sentido de realidad en las propias posibilidades ni se rompan las loables metas de carácter social antes indicadas para la escuela. Aquí se parte, por tanto, del criterio que es función de la escuela básica formar personas competentes, no en el estricto sentido profesional, sino en el sentido amplio de ser capaces de resolver los problemas que plantea la vida cotidiana en sus múltiples facetas de información, relación y compromiso. Y una forma de lograr esta competencia es mediante la actividad colaboradora con los demás, al tiempo que se consolida el hábito de superación hacia uno mismo.

\section{2. ¿POR QUÉ ERA NECESARIO EL CAMBIO QUE CONLLEVAN LAS COMPETENCIAS?}

Hay que insistir que asignar ahora a la escuela la tarea de procurar la adquisición de competencias no es una simple cuestión de cambio terminológico respecto 
a lo que tradicionalmente ha estado haciendo, sino una forma diferente y actual de entender su función educativa. Hay razones de fondo para pedir un cambio en las metas escolares, tradicionalmente vinculadas a la adquisición de conocimientos estrictamente académicos y la adquisición de habilidades que se agotan en el mismo ámbito escolar; algunas de estas razones se comentan a continuación.

Un primer factor a considerar es el constante aumento de los conocimientos, que desde hace prácticamente un siglo hace imposible que el sistema educativo pueda tratarlos todos. Este aumento se ha visto acompañado de una progresiva especialización dentro de la cual se produce la renovación más acelerada. La consecuencia directa para el sistema educativo, en cualquiera de sus niveles, es la exigencia de realizar la selección de los conocimientos que resulten más necesarios para obtener la comprensión básica de cada ámbito y que permitan seguir aprendiendo. Y esta selección se hará en función de su necesidad para comprender y actuar el mundo actual.

El principio ya ampliamente consolidado de "aprendizaje a lo largo de la vida" constituye la expresión no sólo de la posibilidad y deseo de perfeccionamiento continuo de cada persona, sino la respuesta al constante aumento y modificación de los conocimientos vigentes. Estamos "Condenados" a aprender de por vida, como decía Illich. Todo lo que signifique formación inicial cobra, por tanto, especial relevancia y dentro de ella es fundamental la adquisición de los conocimientos y las estrategias de acción que resulten fundamentadoras, básicas, para seguir aprendiendo. Así se explica el comentado principio de "retorno a lo básico".

La revisión curricular no termina con la selección de los contenidos sino que incluye también la perspectiva didáctica que pone el énfasis en la figura del aprendiz y no tanto en la lógica de la docencia. La escuela debe centrarse en los aprendizajes de los alumnos, y la planificación curricular por parte del docente en la estrategia que les haga posible y no en una actividad que se justifique por sí misma. Tradicionalmente, al hablar de currículum y de programas escolares se hacía referencia a los contenidos, las prescripciones legales y la actividad docente, mientras que al hablar de capacidades, de competencias, el énfasis se pone en lo que debe conseguir el sistema educativo en sus alumnos; se cambia el punto de vista en beneficio del alumno y sus posibilidades de aprendizaje.

El crecimiento y la especialización de todos los conocimientos se han visto acompañados de una amplia diversidad de fuentes para su difusión y apoyo; son los propios de la denominada "sociedad de la información". Ante esta realidad, la escuela ha perdido el monopolio del saber, que se encuentra depositado en múltiples fuentes informativas de cada vez más fácil acceso. Pero esta multiplicidad de fuentes no es garantía de aprendizaje sistemático, estructurado, porque la información extraescolar es muy diversa y desorganizada. La escuela, por tanto, sigue siendo la única institución que presenta las informaciones de forma estructurada y secuenciada. Pero hay algo más. El conjunto de fuentes informativas ofrece en numerosas ocasiones contradicciones, además de fines no siempre de acuerdo con los principios morales que rigen la educación escolar. Por consiguiente, a la 
escuela le corresponde no sólo preparar los sujetos para la adecuada selección y organización de las informaciones accesibles a través de la multiplicidad de medios sociales existentes, sino también prepararlos para ejercer sobre ellas la crítica valorativa; en caso contrario es evidente el peligro de caer víctima de la manipulación. Es así que la adquisición de técnicas y hábitos para acceder a la información de varias fuentes, muchas de ellas digitales, son nuevas metas que se le asignan a la escuela de nuestros días, y que ha de incorporar a la pléyade de las que ya le son tradicionales. Ser hoy un ciudadano competente implicar serlo en el dominio básico de las Tic.

La inmensa cantidad de información disponible nos obliga a volver a poner el énfasis en los objetivos curriculares por encima de los contenidos, después de una etapa que habían sido un poco depreciados por estar fuertemente ligados a la tradición conductista, que les había atomizado hasta a un nivel que recordaba las operaciones laborales de naturaleza muy específica. Ahora vuelve la mirada hacia los objetivos educativos, pero con unas características que los alejan de los objetivos conductuales específicos de carácter conductista, porque se trata de objetivos que no se agotan en sí mismos sino que establecen las bases para un perfeccionamiento continuo de las capacidades que conllevan, materializadas en el principio del aprendizaje a lo largo de la vida. Serán precisamente los aprendizajes más específicos los que más rápidamente quedarán obsoletos con el paso del tiempo y los consiguientes cambios sociales y tecnológicos. Las habilidades específicas se agotan en sí mismas y sólo pueden ser consideradas como objetivos de aprendizaje en la medida en que sirvan para concretar las metas más amplias (competencias).

Junto con otras preocupaciones, como las señaladas, el actual sistema educativo tiene una preocupación creciente para conseguir la máxima equidad para sus alumnos, más cuando las desigualdades sociales siguen creciendo. Por ello tiene que preocuparse de determinar metas de aprendizaje que estén al alcance de la inmensa mayoría del alumnado, en la línea de las consideradas como competencias básicas. La síntesis de la equidad con la calidad conseguirá si realmente las competencias identificadas como básicas son relevantes, cumplen la función asignada y se ponen al alcance de la inmensa mayoría de los alumnos. Pero, además, las competencias planteadas deben permitir un avance constante para quienes tienen la posibilidad de hacerlo; es decir, si tienen posibilidad de gradación en su consecución. Los sistemas educativos de calidad son los que garantizan un mínimo fundamental y suficiente para todos y posibilitan un máximo para cada uno.

\section{LOS CALIFICATIVOS SON DECISIVOS}

A menudo se habla de competencias de manera genérica, lo que ayuda poco a aclarar el concepto que debe vincularse con una etapa concreta del sistema educativo. Por ello, el calificativo que las acompañe resulta decisivo para situar las competencias en un nivel determinado y con una finalidad específica. No es lo mismo 
hablar de competencias básicas que de competencias claves o de competencias laborales, etc. La generalización del término ha hecho que las denominaciones se mezclen y los autores llegan a presentar ejemplos de competencias de manera indistinta. Así no es extraño advertir como se mencionan competencias claramente vinculadas al mundo laboral o profesional en un contexto de educación básica, mientras que se muestran competencias claramente básicas -el típico ejemplo de saber leer y escribir- en contextos profesionales universitarios. Todo queda englobado bajo el denominador mágico del término "competencias". Con el afán de intentar una clarificación al respecto, a continuación se presenta una propuesta de clasificación.

Competencias básicas. Se vinculan con el nivel básico de la educación (periodo escolar obligatorio) y se refieren al conjunto de exigencias mínimas que debería adquirir un ciudadano de nuestros tiempos al final de la escolaridad obligatoria, como garantía de pertenencia (calidad) y de equidad del sistema educativo. Incluye competencias curriculares que se vincularían directamente con un área o asignatura del currículum, y otras (transversales) que deberían ser tratadas en todas las materias del currículo, como garantes de una formación integral de la persona.

Las competencias básicas se refieren a los objetivos generales del final de la escolaridad obligatoria, dado que deben estar al alcance de todo el alumnado - casos de dificultades especiales aparte-, y deben servir de base y fundamento para seguir aprendiendo a lo largo de toda la vida, además de asegurar los mínimos necesarios para la comprensión y actuación en el mundo actual por parte de los alumnos que terminan su escolarización básica.

Competencias técnico-profesionales. Con esta denominación se suelen abarcar las competencias vinculadas a la formación profesional correspondiente al nivel de escolarización de la secundaria postobligatoria. En este nivel educativo es donde las competencias arraigaron en primer término, y donde se cuenta con mayor experiencia para su enseñanza y evaluación. Incluirían competencias estrictamente vinculadas a la acción técnica (competencias específicas de una rama técnico-profesional) y competencias también transversales de formación integral de la persona y de adaptación al mundo laboral.

Competencias clave. El término fue introducido por la OCDE en sus evaluaciones del Proyecto PISA y en el estudio conocido como Proyecto DeSeCo del 2003, y se refiere al conjunto de competencias que son necesarias para "una vida exitosa y un buen funcionamiento en la sociedad" (Rychen y Salganik, 2003). Estas competencias pretenden un amplio espectro de aplicabilidad en los países desarrollados y, por tanto, superan las estrictamente curriculares y se desarrollan a lo largo de la vida (Tabla 1). Esta misma denominación ha sido adoptada por la uE para su propuesta competencial. 
TABLA 1. COMPETENCIAS CLAVE PARA EL ÉXITO EN LA VIDA Y EL BUEN FUNCIONAMIENTO DE la sociedad. Proyecto DeSeCo De la ocde (2003)

\begin{tabular}{|c|c|}
\hline $\begin{array}{c}\text { ACTUAR DE MANERA } \\
\text { AUTÓNOMA }\end{array}$ & $\begin{array}{ll}\text { - } & \text { Capacidad de defender y afirmar sus derechos, sus intereses, } \\
\text { sus responsabilidades, sus límites y sus necesidades } \\
\text { - } \\
\text { Capacidad de proyectar y realizar planes } \\
\text { de vida y proyectos personales } \\
\text { - Capacidad de actuar teniendo presente } \\
\text { el conjunto de la situación }\end{array}$ \\
\hline $\begin{array}{l}\text { UTILIZAR RECURSOS DE } \\
\text { MANERA INTERACTIVA }\end{array}$ & $\begin{array}{ll} & \text { Capacidad de utilizar el lenguaje, los símbolos } \\
& \text { y los textos de manera interactiva } \\
\text { - } & \text { Capacidad de utilizar el saber y la } \\
\text { información de manera interactiva } \\
\text { - Capacidad de utilizar la (nueva) } \\
\text { tecnología de manera interactiva }\end{array}$ \\
\hline $\begin{array}{c}\text { ACTUAR EN LOS } \\
\text { GRUPOS SOCIALMENTE } \\
\text { HETEROGÉNEOS }\end{array}$ & $\begin{array}{l}\text { - Capacidad de establecer buenas relaciones con los demás } \\
\text { - Capacidad de cooperar } \\
\text { - } \quad \text { Capacidad de controlar y resolver conflictos }\end{array}$ \\
\hline
\end{tabular}

Competencias profesionales. Por competencias profesionales estrictas habría que entender las que corresponden a un profesional de nivel superior, y que suponen la capacidad de resolver problemas profesionales complejos mediante conocimientos científicos y habilidades técnicas, aplicadas con los planteamientos éticos propios de la profesión en cuestión. Estas competencias han servido de justificación para las actuales reformas de las carreras universitarias. Se distingue entre competencias genéricas, que serían propias de todo titulado universitario, y especificas, que corresponderían a una carrera en concreto. Las competencias propuestas en el Proyecto Tuning (2004) serían de las primeras.

\section{Características de las COMPETENCIAS BÁSiCAS}

Generalmente, las competencias básicas se proponen para ser alcanzadas al final de la escolarización, a la que se llega tras un alcance secuencial entre la escolarización primaria y la secundaria. Pero dentro de la consecución de cada competencia existe la posibilidad de gradación, es decir, de alcanzar diferente nivel de dominio de la misma, con lo cual se puede establecer desde un nivel mínimo hasta un nivel máximo o de excelencia. Así lo ha establecido, por ejemplo, el Programa PISA, que en sus competencias matemáticas tenía una gradación de seis niveles de dificultad, tal como se muestra en el cuadro adjunto. 


\begin{tabular}{|c|c|}
\hline Nive & \\
\hline 6 & $\begin{array}{l}\text { En el nivel } 6 \text { los alumnos saben formar conceptos, generalizar y utilizar información bas.ada en } \\
\text { investigaciones y modelos de situaciones de problemas complejos. Pueden relacionar diferentes } \\
\text { fuentes de información y representaciones y traducirlas entre ellas de manera flexible. Los } \\
\text { estudiantes de este nivel poseen un pensamiento y razonamiento matemático avanzado. Estos } \\
\text { alumnos pueden aplicar su entendimiento y comprensión, asi como su dominio de las operaciones } \\
\text { y relaciones matemáticas simbólicas y formales y desarrollar nuewos enfoques y estrategias para } \\
\text { abordar situaciones nuevas. Los alumnos pertenecientes a este nivel pueden formular y comunicar } \\
\text { con exactitud sus acciones y reflexiones relativas a sus descubrimientos, interpretaciones, } \\
\text { argumentos y su adecuación a las situaciones originales. }\end{array}$ \\
\hline 5 & $\begin{array}{l}\text { En el nivel } 5 \text {, los alumnos saben desarrollar modelos y trabajar con ellos en situaciones complejas, } \\
\text { identificando los condicionantes y especificando los supuestos. Pueden seleccionar, comparar y } \\
\text { evaluar estrategias adecuadas de solución de problemas para abordar problemas complejos relativos } \\
\text { a estos modelos. Los alumnos. pertenecientes a este nivel pueden trabajar estratëgicamente } \\
\text { utilizando habilidades de pensamiento y razonamiento bien desarrolladas, así como representaciones } \\
\text { adecuadamente relacionadas, caracterizaciones simbólicas y formales, e intuiciones relativas a estas } \\
\text { situaciones. Pueden reflexionar sobre sus acciones y formular y comunicar sus interpretaciones y y } \\
\text { razonamientos. }\end{array}$ \\
\hline 4 & $\begin{array}{l}\text { En el nivel } 4 \text {, los alumnos pueden trabajar con eficacia con modelos explíitos en situaciones } \\
\text { complejas y concretas que pueden conllevar condicionantes o exigir la formulación de supuestos. } \\
\text { Pueden seleccionar e integrar diferentes representaciones, incluidas las simbólicas, asociándolas } \\
\text { directamente a situaciones del mundo real. Los alumnos de este nivel saben utilizar habilidades bien } \\
\text { desarrolladas y razonar con flexibilidad y con cierta perspicacia en estos contextos. Pueden elaborar y } \\
\text { comunicar explicaciones y argumentos basados en sus interpretaciones, argumentos y acciones. }\end{array}$ \\
\hline 3 & $\begin{array}{l}\text { En el nivel 3, los alumnos saben ejecutar procedimientos descritos con claridad, incluyendo aquellos } \\
\text { que requieren decisiones secuenciales. Pueden seleccionar y aplicar estrategias de solución de } \\
\text { problemas sencillos. Los alumnos de este nivel saben interpretar y utilizar representaciones basadas } \\
\text { en diferentes fuentes de información y razonar directamente a partir de ellas. Son también capaces } \\
\text { de elaborar breves escritos exponiendo sus interpretaciones, resultados y razonamientos. }\end{array}$ \\
\hline 2 & $\begin{array}{l}\text { En el nivel } 2 \text {, los alumnos saben interpretar y reconocer situaciones en contextos que solo requieren una } \\
\text { inferencia directa. Saben extraer información pertinente de una sola fuente y hacer uso de un único } \\
\text { modelo representacional. Los alumnos de este nivel pueden utilizar algoritmos, fómulas, } \\
\text { procedimientos o convenciones elementales. Son capaces de efectuar razonamientos directos e } \\
\text { interpretaciones literales de los resultados. }\end{array}$ \\
\hline 1 & $\begin{array}{l}\text { En el nivel 1, los alumnos saben responder a preguntas relacionadas con contextos que les son } \\
\text { conocidos, en los que está presente toda la información pertinente y las preguntas están claramente } \\
\text { definidas. Son capaces de identificar la información y llevar a cabo procedimientos rutinarios siguiendo } \\
\text { unas instrucciones directas en situaciones explicitas. Pueden realizar acciones obvias que se deducen } \\
\text { inmediatamente de los estimulos presentados. }\end{array}$ \\
\hline
\end{tabular}


El número de grados o niveles que se formulen para cada competencia es una decisión que dependerá del uso que de tales grados se quiera hacer. Lógicamente, al aumentar el número de grados aumenta la complejidad de la competencia. Dos son los sistemas básicos que se utilizan para realizar tal gradación: el estadístico y el criterial, si bien caben combinaciones de ambos. Mediante el sistema estadístico se realizan los grados en función de los cortes que se establezcan en los resultados ya obtenidos por los destinatarios de las pruebas de competencia. Mediante el sistema criterial, los grados se establecen en función de la opinión de posibles expertos sobre la progresiva dificultad en el dominio de la competencia; en este caso se suele recurrir a indicadores de logro para establecer tales grados. El Programa PISA establece sus niveles de acuerdo a criterios estadísticos vinculados a los ajustes que proporciona la "teoría de la respuesta al ítem" (TRI), mientras que la propuesta del Departamento de Enseñanza de la Generalidad de Cataluña se fundamenta en criterios de dificultad establecidos por las comisiones técnicas encargadas de identificar y desarrollar las competencias básicas del currículo.

Aún otra consideración importante. En general, las competencias sólo se pueden materializar plenamente en el ámbito real (cotidiano, profesional...), mientras que el sistema educativo o formativo lo que puede conseguir son las capacidades que harán posible la pretendida actuación competente (Mastache, 2007). El logro de las competencias se alcanzará después de la consecución de las capacidades que conllevan. Por lo tanto, las competencias son susceptibles de ser analizadas en función de las capacidades que ponen en juego. Siguiendo con el mismo ejemplo del Programa PISA, se puede advertir qué capacidades se considera que intervienen en las competencias matemáticas. Las siete capacidades matemáticas fundamentales utilizadas en este marco son las siguientes:

- Comunicación

- Matematización

- Representación

- Razonamiento y argumentación

- Diseño de estrategias para resolver problemas

- Utilización de operaciones y lenguaje simbólico, formal y técnico

- Utilización de herramientas matemáticas

Las capacidades que intervienen en las competencias se pueden considerar como requisitos potenciales de estas, algunas son específicas de las competencias en cuestión, otras tienen un carácter transversal y se aplican a diversos ámbitos competenciales. Del listado mencionado podríamos considerar que la comunicación pertenece al segundo tipo, mientras que la matematización sería del primero. Estas consideraciones son una constatación de la perspectiva interdisciplinaria o transversal que tienen en conjunto las competencias. Hay que advertir, sin embargo, que los análisis de los elementos que tienen las competencias no deberían llevar a una atomización excesiva que las hiciera perder su amplitud y funcionalidad didáctica, porque es fácil acabar formulando subapartados o subcompetencias, 
hasta llegar a situaciones que recordarán los listados de objetivos específicos del conductismo.

\section{Tradiciones y nOVEDAdes EN LaS COMPETENCIAS BÁSicas}

Las competencias recogen aspectos del proceso de enseñanza-aprendizaje que ya habían entrado con fuerza en el ámbito pedagógico unos decenios atrás, otros suponen una novedad o avance. Por tanto, no se debe considerar a las competencias como un elemento de ruptura respecto al pasado inmediato porque, si así fuera, seguramente constituirían una novedad efímera; su mejor garantía de permanencia es que se sitúan en una línea de continuidad con el pasado inmediato. Entre los principales elementos de continuidad se pueden señalar los siguientes:

a) Insisten en la actividad del alumno, como ya hizo el movimiento de la Escuela Nueva y destacaron autores como John Dewey (learning by doing). Las competencias enfatizan la actividad como medida para aprender y manifiestan la utilidad de los aprendizajes, sin excluir por ello los aprendizajes conceptuales, logrados mediante la atención y reflexión. El concepto de actividad debe entenderse en sentido amplio, de manera que la mayoría de las actividades propuestas serán de tipo mental.

b) En la línea de lo anterior, las competencias incluyen conocimientos, habilidades y actitudes, porque no se trata de un simple saber hacer sin comprensión de la actividad, sino de un saber hacer razonado y con compromiso (aprendizaje situado) por parte de quien lo realiza. Algunas de las competencias más transversales, como por ejemplo: "adoptar posicionamientos personales críticos ante los acontecimientos sociales" o "respetar la diversidad cultural en el marco de las sociedades democráticas", pueden ser consideradas como equivalentes a los objetivos actitudinales que hace tiempo se propone la escuela junto a los estrictamente cognitivos. Esto sin olvidar que todo logro actitudinal pasa siempre por adquirir conocimientos razonados y realizar prácticas coherentes.

c) Pretenden la globalización de los aprendizajes, viejo principio pedagógico que tiene amplio arraigo en la educación infantil y que va desapareciendo a medida que se avanza en las etapas del sistema educativo. Las competencias nos vuelven a pedir el esfuerzo de integrar aprendizajes que pertenecen a áreas o materias escolares diversas, porque los problemas complejos que pretender resolver necesitan de la contribución interdisciplinar de las informaciones y aplicaciones. La capacidad de integración de todos los aprendizajes logrados en las diversas materias escolares es una muestra de logro competencial.

d) Piden aprendizajes significativos, integrados con los anteriores que el sujeto ya posee, como garantía de firmeza y comprensión de los nuevos, así como del necesario factor acumulativo de los mismos para poder avanzar 
en los grados de dominio de las competencias. Para llegar a la excelencia en una competencia hay que pasar por fases sucesivas que fundamenten los avances.

e) Las competencias parten de la necesidad de la transferencia, porque una formación nunca puede preparar para todas las situaciones que el sujeto se puede encontrar en el presente y en el futuro. La transferencia obliga a generalizar a partir de las situaciones concretas para poder atender otras situaciones similares, no idénticas, hasta llegar a la capacidad de innovación, que superará los modelos previos. Transferencia y creatividad son conceptos pedagógicos ya tradicionales en muchos ámbitos y que en los aprendizajes de naturaleza competencial son imprescindibles. Una persona competente en un ámbito resuelve situaciones diversas y no se limita a repetir soluciones rutinarias o simplemente consolidadas.

Pero las competencias también aportan algunas novedades, que permiten pensar que se avanza en el proceso de mejora de la tarea pedagógica. Estas aportaciones se podrían resumir en los siguientes apartados:

a) Vinculan la escuela con la vida real, rompiendo la tendencia a hacer de la escuela un mundo aparte de aquel para el que ha de preparar. Ciertamente, todo el mundo real no debe penetrar en el sistema educativo, porque no todo es compatible con los valores que éste quiere fomentar, pero hay que tenerlo presente para garantizar que se prepara a los niños y jóvenes para ser ciudadanos conscientes y responsables en el mundo que les tocará vivir. Este criterio lleva, por ejemplo, a la necesidad de la constante puesta al día de los contenidos, para garantizar el principio de realismo, así como procurar vincular las actividades de aprendizaje con las situaciones de la vida real y cotidiana de los alumnos. No hará falta insistir en que la puesta al día no excluye el mantenimiento de contenidos históricos y de permanente actualidad.

b) Determinan mínimos equitativos para todo el alumnado, superando la simple clasificación de los alumnos en función de aprendizajes estrictamente académicos, cumpliendo así el encargo de preparar a todos para la sociedad a la que pertenecen; de otro modo, las competencias tratadas no merecerían el calificativo de «básicas” y, consecuentemente, tampoco tendría justificación la obligatoriedad de la enseñanza hasta una determinada edad. La equidad, sin embargo, no debe ser obstáculo para que unos alumnos puedan avanzar más que otros en razón de su capacidad y esfuerzo, lo que lleva a la dimensión de la calidad en el sistema educativo, entendida como la posibilidad de que cada uno pueda desarrollarse al máximo de su potencial.

c) Introducen criterios de eficacia y eficiencia, términos que vienen del mundo económico, pero que aquí debemos interpretarlos como la verificación de que las situaciones o problemas a los que se aplican las competencias son efectivamente resueltos, y lo son por la vía más eficiente, es 
decir, por la vía que supone optimizar los recursos disponibles. El aprendizaje en base a competencias se preocupa de valorar varias alternativas posibles de solución y elige la mejor; se trata de la teoría de resolución de problemas aplicada de manera generalizada. Esto es lo que hace toda persona competente en su ámbito de actuación.

d) Los procesos de enseñanza-aprendizaje de carácter competencial exigen evaluaciones interdisciplinarias, como consecuencia lógica del aprendizaje globalizador e interdisciplinar que plantean. Si siempre ha sido necesario que el profesorado planificara de forma conjunta el currículo, ahora resulta imprescindible para poder acometer los aprendizajes desde diversos ámbitos y, consecuentemente, evaluar de manera conjunta, rompiendo así una larga tradición, arraigada en todos los niveles del sistema educativo, de hacer evaluaciones estrictamente vinculadas a una materia o área de conocimiento. Esto no excluye que haya competencias que se adscriban plenamente a un ámbito disciplinar concreto, pero muchas deben ser acometidas desde más de uno de ellos; a título de ejemplo de estas últimas se pueden citar las competencias lingüísticas o las vinculadas al uso de las tecnologías digitales.

e) Las competencias son objetivos amplios, a los que se podrá llegar mediante un proceso progresivo de aproximación. Para ello, será útil analizar los componentes que integran cada competencia y considerar el logro de cada uno de ellos, previamente a la síntesis que supone la competencia en cuestión. Hay que evitar obsesionarse en todo momento para estar "enseñando competencias"; éstas deben ser el referente final, no cada fase intermedia del aprendizaje escolar. Cotidianamente, en la actividad escolar se van logrando objetivos específicos, concretos, y otros más amplios, que deben tener las competencias como referentes, como hitos de final de etapa. De la misma manera que se puede afirmar que las competencias son un tipo de objetivos de aprendizaje de carácter amplio, se puede afirmar igualmente que no todos los objetivos de aprendizaje son de carácter competencial.

\section{El marco estatal de las COMPETENCias básicas}

El concepto de competencias entra en el ámbito estatal cuando se hace la primera evaluación internacional del programa PISA de la OCDE, en el año 2000. Esta evaluación no es de carácter curricular sino que se realiza en base a lo que la OCDE denomina "competencias clave". A partir de ese momento, el concepto de competencia entra de manera generalizada en los sistemas educativos no universitarios de los países avanzados, al margen de su existencia ya instalada en la formación profesional, para dar respuesta al desafío que los resultados del PISA plantean a los gobiernos de turno. En el caso de España, además, se añadía la clara voluntad de 
hacer olvidar la LOGSE, a la que se acusó de ser la responsable del bajo rendimiento en las pruebas internacionales.

Las leyes que sustituyeron a la LOGSE, la Ley Orgánica de la Calidad de la Educación (LOCE) promulgada en el 2003, la Ley Orgánica de la Educación (LOE) implantada en el 2006 y la Ley Orgánica de la Mejora de la Calidad de la Educación (LOMCE) del 2013, efectivamente, han supuesto una vuelta a algunos planteamientos anteriores a los años noventa, desapareciendo las propuestas pedagógicas más avanzadas que había en la LOGSE, aunque los textos legales adoptaran conceptos como "calidad", "equidad", "Competencias"... Sin duda los motivos han sido más políticos que pedagógicos: cambios de partido en el Gobierno central y la obsesión por eliminar las propuestas de una ley (LOGSE) considerada culpable de todos los males del sistema educativo.

En los currícula derivados de la LOCE no aparecieron las competencias básicas. Tanto en los currículos publicados a raíz de la LOE como en los de la LOMCE, las competencias básicas aparecen de alguna manera, pero en el primer caso en un anexo, mientras que en el caso de la LOMCE sólo se mencionan en su inicio, si bien luego son objeto de desarrollo en una orden posterior. En ambos casos, sin embargo, el desarrollo curricular se realizó al margen de las competencias básicas. Está claro que los redactores de estos currículos básicos de primaria y secundaria no estaban familiarizados con las competencias, y simplemente se añadieron después, dejando bajo la responsabilidad de los centros escolares su desarrollo y correspondiente inserción en el currículo. Las "Competencias" a las que hacen referencia los currículums estatales son las recomendadas por la Unión Europea en el 2006, a partir de un trabajo realizado por un grupo de trabajo en el 2004, que las calificaban como "competencias clave", precisamente porque no tienen carácter curricular y se proponían para el conjunto de los países de la UE (Grupo de Trabajo B, 2004). Con todo, el Ministerio de Educación hizo unos cambios a la propuesta original que son indicativos de sus preocupaciones ideológicas en cada caso (Tabla 2).

Como se puede advertir, en ambos casos de la propuesta española desaparece la primera "competencia" dirigida a la comunicación en lengua materna y se efectúan algunos cambios de denominación en las restantes. El currículo de la LOMCE vuelve a juntar matemáticas y ciencias y tecnología, que se habían separado en el de la LOE. En todo caso, hay que advertir que en la última propuesta curricular las competencias en cuestión ya no son calificadas como "básicas", aunque esta denominación es la que figura en la ley.

Este listado no puede considerarse una relación de competencias básicas por dos razones. La primera porque no tienen carácter curricular, es decir, que no se vinculan directamente con ningún currículum concreto, ni se fija el nivel de la etapa educativa a la que se dirigen (aunque hay que suponer que es al conjunto de la educación obligatoria). Y en segundo término porque su formulación, en forma de un simple enunciado, no concreta los elementos sobre los que se aplican ni se especifica su nivel de logro. Por tanto, se trata más bien de "bloques competenciales" de carácter amplio, que piden ser especificados y determinados en su 
alcance. Esto no excluye, sin embargo, que las explicaciones que acompañan a esta relación de bloques competenciales no sean muy útiles para el desarrollo que se pueda hacer de los mismos.

TABla 2. COMPARACión ENTRE LAS COMPETENCIAS PROPUESTAS POR UN GRUPO

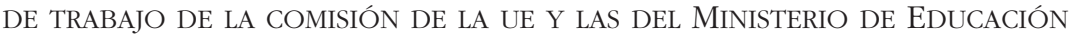

\begin{tabular}{|c|c|c|}
\hline $\begin{array}{l}\text { LAS COMPETENCIAS CLAVE } \\
\text { RECOMENDADAS POR LA UE } \\
(2006)\end{array}$ & $\begin{array}{l}\text { LAS COMPETENCIAS BÁSICAS } \\
\text { DEL CURRÍCULUM DE LA LOE } \\
\text { (2006) }\end{array}$ & $\begin{array}{c}\text { LAS COMPETENCIAS DEL } \\
\text { CURRÍCULUM DE LA LOMCE } \\
(\text { RD 03/01/2014) }\end{array}$ \\
\hline $\begin{array}{l}\text { 1. Comunicación en la } \\
\text { lengua materna } \\
\text { 2. Comunicación en una } \\
\text { lengua extranjera } \\
\text { 3. Competencia matemática } \\
\text { y competencias básicas } \\
\text { en ciencia y tecnología } \\
\text { 4. Competencia digital } \\
\text { 5. Aprender a aprender } \\
\text { 6. Competencias } \\
\text { interpersonales y cívicas } \\
\text { 7. Espíritu emprendedor } \\
\text { 8. Expresión cultural }\end{array}$ & $\begin{array}{l}\text { 1. Competencia en } \\
\text { comunicación lingüística } \\
\text { 2. Competencia matemática } \\
\text { 3. Competencia en el } \\
\text { conocimiento y la interacción } \\
\text { con el mundo físico } \\
\text { 4. Tratamiento de la información } \\
\text { y competencia digital } \\
\text { 5. Competencia social } \\
\text { y ciudadana } \\
\text { 6. Competencia cultural } \\
\text { y artística } \\
\text { 7. Competencia para } \\
\text { aprender a aprender } \\
\text { 8. Autonomía e iniciativa } \\
\text { personal }\end{array}$ & $\begin{array}{l}\text { 1. Comunicación lingüística } \\
\text { 2. Competencia matemática } \\
\text { y competencias básicas } \\
\text { en ciencia y tecnología } \\
\text { 3. Competencia digital } \\
\text { 4. Aprender a aprender } \\
\text { 5. Competencias } \\
\text { sociales y cívicas } \\
\text { 6. Sentido de iniciativa y } \\
\text { espíritu emprendedor } \\
\text { 7. Conciencia y expresiones } \\
\text { culturales }\end{array}$ \\
\hline
\end{tabular}

\section{El DESARROLLO DE LAS COMPETENCIAS BÁSICAS CURRICULARES REALIZADO EN CATALUÑA}

El Departamento de Enseñanza de la Generalidad de Cataluña ha hecho un despliegue de las competencias básicas que considera adecuadas de ser alcanzadas por el alumnado catalán al final de las etapas de educación primaria y de educación secundaria obligatoria. Estas competencias se han vinculado con las respectivas áreas curriculares y asignaturas, bajo la consideración de objetivos finales de etapa.

En Cataluña había antecedentes de haber trabajado en competencias básicas propias, si bien con el paso del tiempo el concepto se ha ido afinando, tanto por reflexión propia como por los avances que en este terreno han hecho otros países. Se puede recordar, por ejemplo, un estudio realizado en 1999, en colaboración con los territorios de las Islas Baleares y Canarias, en el marco de la FREREF, Fundación Regional Europea (Departament d'Ensenyament, 2000; Sarramona, 2000). A partir de este estudio, en el 2001 se iniciaron las primeras evaluaciones en competencias básicas aplicadas a todos los alumnos de cuarto de primaria y segundo de ESO, que se han seguido realizando posteriormente, y que han sido un ejemplo 
para otros territorios del Estado, como las Islas Canarias, Andalucía, Castilla-La Mancha, etc., y que luego recogió el Gobierno central, poniendo la evaluación en competencias básicas en la LOCE (2003) primero y la LOE después (2006). Dentro de estos antecedentes se puede mencionar el Congreso Internacional que sobre competencias básicas organizó el Departamento de Enseñanza en el 2003 (Departament d'Ensenyament, 2003a), donde se mostró públicamente la nueva propuesta en competencias básicas curriculares elaborada dentro de la Conferencia Nacional de Educación (2002).

Posteriormente hubo unos años de estancamiento en el trabajo por competencias tal como se habían entendido en Cataluña, en beneficio de la propuesta surgida del Ministerio con la LOE, y que ya ha sido comentada anteriormente. Ha sido a partir de 2012 que el Departamento de Enseñanza ha recuperado la línea de considerar las competencias básicas como objetivos curriculares vinculados a las áreas y materias respectivas, organizando un conjunto de grupos de trabajo encargados de identificarlas y desarrollarlas mediante orientaciones didácticas para los docentes. El trabajo resultante ha sido fiel al concepto internacionalmente aceptado de competencia básica y supone un claro avance respecto a los anteriores. Las fuentes utilizadas para determinar tales competencias básicas curriculares han sido:

- La interpretación en visión competencial de los objetivos curriculares del entonces currículum vigente surgido de la LOE, pues no se pretendía inicialmente hacer un currículum nuevo sino añadir los objetivos competenciales que guiaran la actividad docente en la línea seguida por los países avanzados. A tal efecto se tuvieron en cuenta especialmente los contenidos que debían soportar las competencias.

- Los estudios anteriormente realizados en Cataluña, tanto en el seno del Departamento de Enseñanza como a cargo de profesionales que han trabajado en la universidad o en centros escolares.

- Los currícula de otros países que han incorporado las competencias básicas y han desarrollado estudios al respecto.

- Los documentos publicados por la OCDE vinculados al programa PISA.

- Y, de manera preferente, las aportaciones que los miembros de los respectivos grupos de trabajo han hecho. En este sentido se ha de considerar que ha sido un buen ejemplo de construcción social de conocimiento, ya que las experiencias y sugerencias personales pasaban siempre por la valoración colectiva, de manera que el resultado final ha sido fruto del consenso. Para los miembros de las comisiones ha sido también una situación de aprendizaje en una tarea de la que no había antecedentes en la línea fijada. La identificación y desarrollo de las competencias básicas que nos ocupan han adoptado la estructura que se presenta gráficamente en la Figura 1 y que se detalla a continuación. 
FiguRA 1. ESTRUCTURA DE LOS DOCUMENTOS SOBRE COMPETENCIAS BÁSICAS PUBLICADOS por el Departamento de Enseñanza de la Generalitat de Cataluña

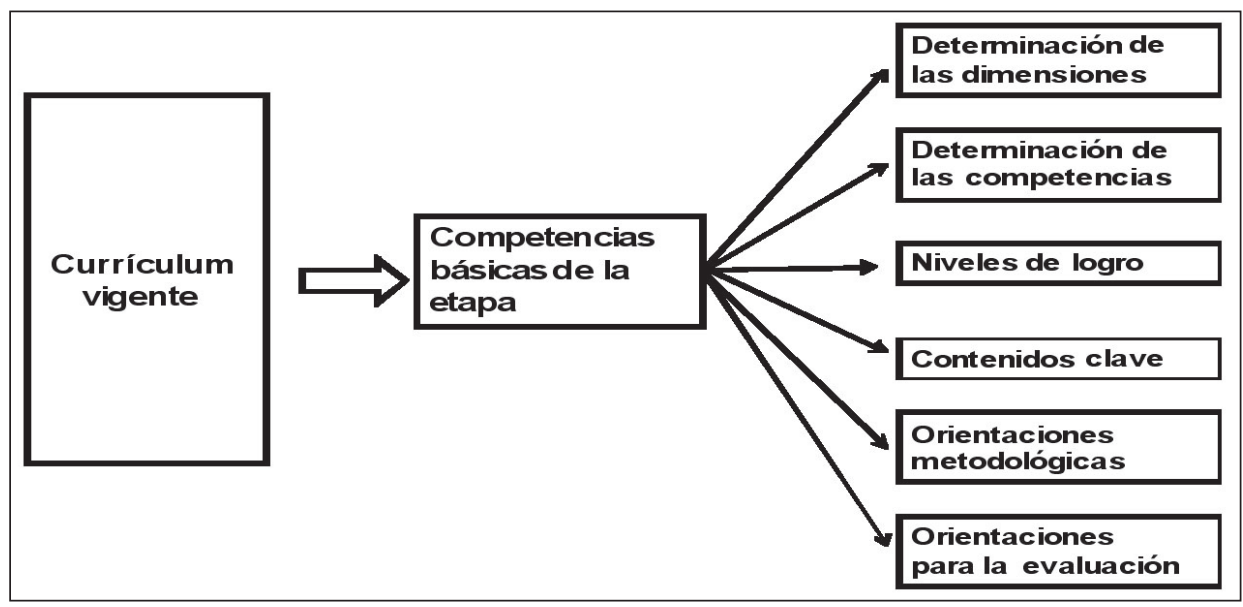

Las competencias están agrupadas en bloques, denominados "dimensiones". Era así como se denominaban los apartados del currículum derivado de la LOE y también los bloques de competencias determinadas hasta el 2003. En todo caso, se trata de una denominación que no tiene otra funcionalidad que agrupar las competencias siguiendo una cierta lógica, epistemológica en unos casos, como en matemáticas, y funcional en otros, como en el caso del ámbito científico. Las dimensiones determinadas son descritas con respecto a su contenido y las competencias que abarcan, como ejemplo, la mostrada en el Cuadro 2.

Dentro de cada dimensión se han determinado las competencias básicas correspondientes. Estas competencias han tenido presente el concepto que se ha señalado anteriormente, haciendo énfasis en su aplicabilidad a la vida real, si bien en algunos casos se ha considerado la perspectiva más académica, en tanto eran una necesidad para un aprendizaje amplio de la materia. El listado total de las competencias vinculadas a las materias y áreas curriculares ha sido moderado, teniendo en cuenta su sentido globalizador, oscilando entre nueve y quince.

El listado de las competencias determinadas para cada ámbito, paralelamente a lo dicho respecto a las dimensiones, se justifica en unos casos por razones epistemológicas, en otros por razones más funcionales - por ejemplo, el caso de las correspondientes a la expresión escrita de primaria- y en otros para poner el énfasis en la importancia actual del tema abarcado, como sería el caso de las vinculadas a la dimensión de salud en la ESO. 
CuAdro 2. Fragmento de la EXPLICACión DE UNA DIMENSIÓN CORRESPONDIENTE A LAS COMPETENCIAS BÁSICAS CIENTÍFICO-TECNOLÓGICAS DEL NIVEL DE ESO

Aquesta dimensió agrupa les competències relacionades amb la intervenció en el món amb recursos tecnològics i les aplicacions de la tecnologia en la indústria i en la vida quotidiana.

Al llarg del dia es fa ús d'un elevat nombre d'objectes resultants de l'acció tecnològica, que proporcionen cobertura a unes necessitats personals, laborals o socials, mitjançant processos que tenen lloc in situ (a casa, a l'escola, al carrer...) o lluny (en una fàbrica, en una central elèctrica...). Una educació escolar bàsica ha de permetre fer-ne ús amb saviesa, coneixent tant les bases del seu funcionament i manteniment, com els riscos personals i mediambientals que poden comportar. Finalment se n'ha de fer ús per desenvolupar la capacitat d'enginyar i de posar a punt eines, instruments, aparells, sistemes o processos que donin resposta a una necessitat.

S'entén per objecte tecnològic qualsevol resultat de factura tecnològica d'origen humà, que sigui tema d'estudi per a l'anàlisi o per al disseny i construcció. En canvi, s'entén per sistema tecnològic una instal·lació complexa d'abast industrial.

L'activitat cientificotecnològica en l'àmbit escolar permet aproximarnos a l'activitat professional amb l'anàlisi d'objectes o de sistemes tecnològics reals, el funcionament dels quals té una base científica emmarcada en els grans principis de la ciència. Sovint l'enginy tecnològic se serveix dels coneixements científics per construir un nou objecte, però també és cert que el disseny de nou instrumental afavoreix el sorgiment de nous coneixements científics; hi ha una sinergia clara entre el treball científic i el tecnològic. Aprendre tecnologia comporta elaborar models i estructures mentals pròpies d'aquest camp, i també comunicar.

Fuente: DePartamento D'EnSENYAMENT, 2013a, 8. 


\section{CUADRO 3. RELACIÓN DE DIMENSIONES Y COMPETENCIAS BÁSICAS DE MATEMÁTICAS DE ESO}

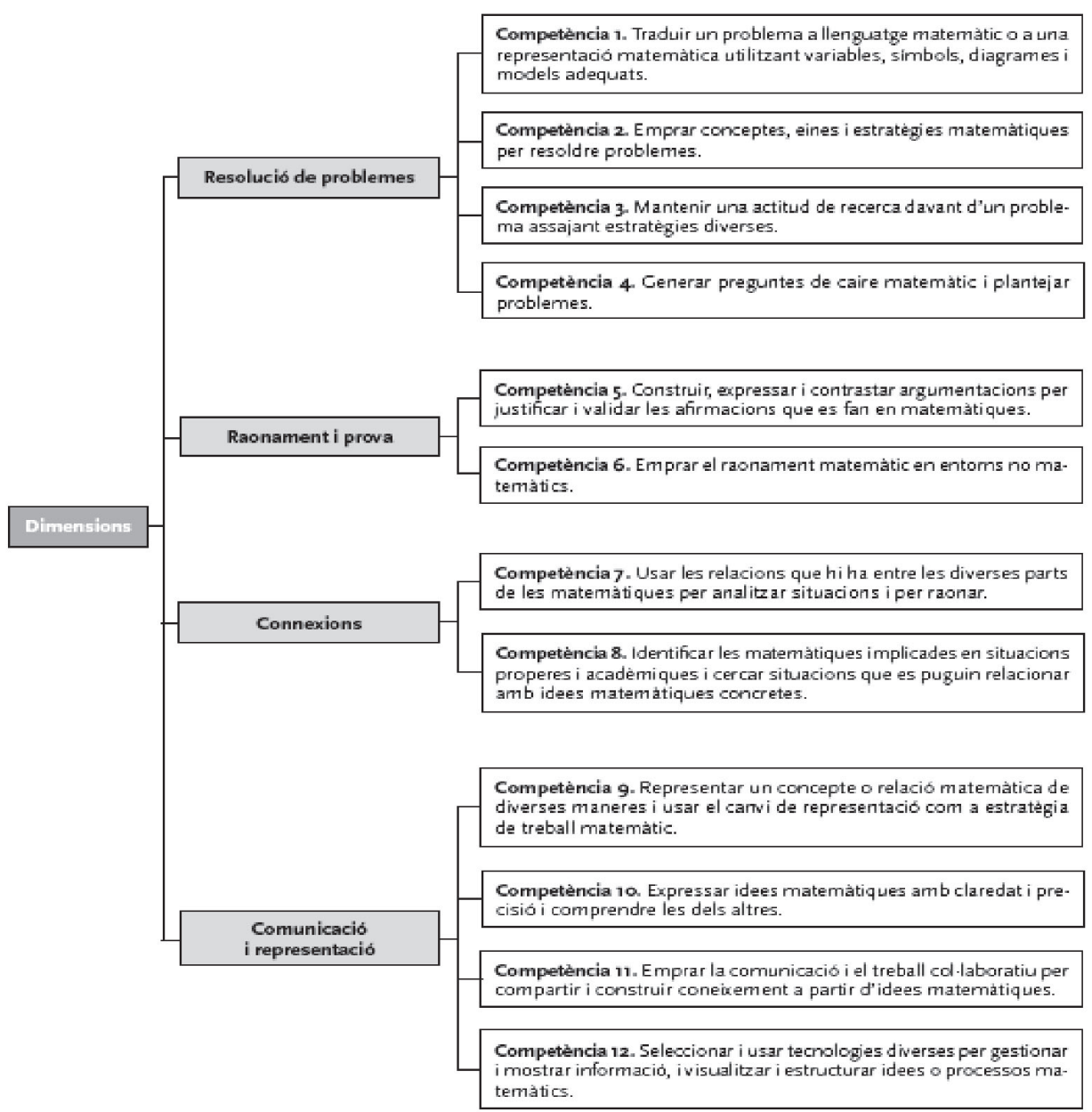

Fuente: DePartamento D'EnSENyament, 2013a, 8.

El enunciado general de cada competencia se concreta en tres niveles de gradación, para señalar las posibilidades de un aprendizaje que vaya desde el nivel mínimo aceptable hasta el nivel considerado excelente, en relación a la etapa educativa. Estos niveles han sido fijados por los grupos de trabajo que han determinado las competencias, y es posible que en el futuro deban ser revisados en función de las informaciones resultantes de su aplicación. En todo caso, siempre 
será un referente inicial para los docentes a la hora de evaluar el nivel de dominio de la competencia, además de los criterios que los centros puedan establecer, pero sin olvidar que estos niveles servirán también para las correspondientes evaluaciones externas que haga el mismo Departamento de Enseñanza.

Las competencias necesitan de informaciones, contenidos, para poder ser alcanzadas. No se pueden comprender situaciones complejas ni resolver problemas sin disponer de informaciones pertinentes. Por tanto, a cada competencia se han vinculado una serie de contenidos considerados "claves" para poder alcanzarla; no se han especificado ni detallado todos los contenidos posibles sino aquellos que resultan importantes para la competencia en cuestión. Esto sin excluir que un mismo contenido puede y debe aparecer en más de una competencia, como consecuencia del carácter transversal que tienen las competencias en muchos casos.

Los contenidos claves han sido determinados de acuerdo a las exigencias de la competencia, pero también teniendo en cuenta el currículum vigente en Cataluña en el momento que se fijaban las competencias, más las aportaciones que las respectivas comisiones consideraron necesarias. Cabe señalar que el concepto de contenido aquí adoptado es el de más tradición académica, es decir, una información que es necesario interiorizar, comprender, para poder actuar.

\section{CUADRO 4. EXPLICACIÓN DE UNA COMPETENCIA DE MATEMÁTICAS DE NIVEL PRIMARIO, CON SU GRADACIÓN Y LOS CONTENIDOS CLAVES A ELLA VINCULADA}

\section{Donar i comprovar la solució d'un problema d'acord amb les preguntes plantejades}

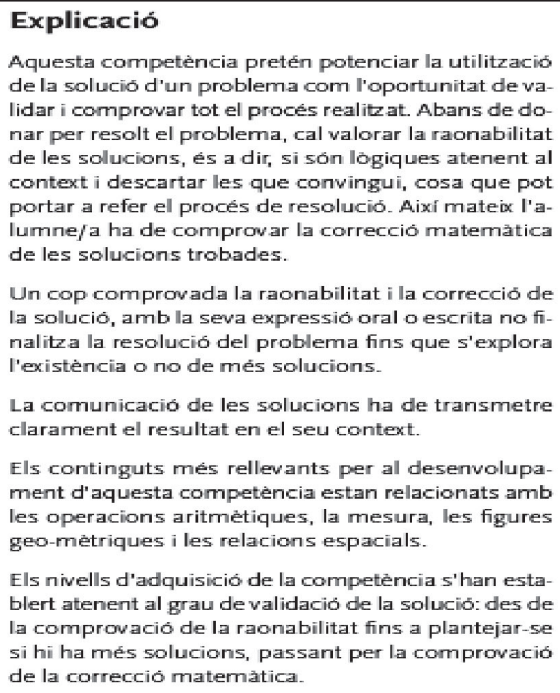

Aquesta competència pretén potenciar la utilitzacio de la solució d'un problema com l'oportunitat de validar i comprovar tot el proces realitzat. Abans de donar per resolt el problema, cal valorar la raonabilitat de les solucions, és a dir, si són lögiques atenent al context i descartar les que convingui, cosa que pot portar a refer ell procés de resolució. Així matebx l'alumne/a ha de comprovar la correcció matemática de les solucions trobades.

Un cop comprovada la raonabilitat i la correcció de la solucio, amb la seva expressio oral o escrita no finalitza la resolució del problema fins que s'explora l'existència o no de més solucions.

La comunicacio de les solucions ha de transmetre clarament el resultat en el seu context.

Els continguts mes rellevants per al desenvolupa ment d'aquesta competencia estan relacionats amb les operacions aritmétiques, la mesura, les figures geo-mètriques i les relacions espacials.

Els nivells d'adquisició de la competència s' han establert atenent al grau de validació de la solucio: ides de la comprowació de la raonabilitat fins a plantejar-se si hi ha més solucions, passant per la comprovació de la correcció matemática.

\section{Gradació}

2.1. Donar la solució d'un problema interpretant la pregunta en el seu context i valorar si la resposta es raonable.

z.z. Donar la solució d'un problema interpretant la pregunta en el seu context, i comprovar tant si es raonable com si compleix les condicions donades.

2.3. Donar la solució d'un problema i comprovar tant si es raonable com correcta, i plantejar-se i explorar si hi pot haver mes solucions.

\section{Continguts clau}

- Nombres. Relacions entre nombres.

- Significat de les operacions, de les propietats i les seves relacions entre elles.

- Calcul (mental, estimatiu, algorísmic, amb eines TIC).

- Magnituds mesurables. Unitats estandards.

- Relacions espacials.

- Les figures geomètriques: elements, caracteristiques ( $2 \mathrm{D}$ i $3 \mathrm{D})$ i propietats.

- Transformacions geomètriques.

Fuente: DePartamento D'EnSENyament, 2013a, 8. 
La novedad que comportan las competencias no reside solamente en su utilización en la vida real, como se ha reiterado, sino también en la metodología didáctica a emplear para su consecución. Para facilitar la aplicación de la metodología didáctica pertinente, cada competencia determinada ha sido acompañada de unas orientaciones metodológicas, unas de carácter muy transversal, aplicables a varias competencias, otras más específicas para la competencia concreta. Estas orientaciones pueden resultar especialmente interesantes si se quiere hacer el viraje metodológico que conllevan las competencias.

\section{CUADRO 5. EJEMPLO DE ORIENTACIONES METODOLÓGICAS VINCULADAS A UNA COMPETENCIA DE MATEMÁTICAS DE NIVEL PRIMARIO}

DIMEN SIO RESOLUCIO DE FROELEMES. COMPETENCIA 1

\section{Orientacions metodològiques}

Resoldre problemes es essencial per a l'aprene ntatge de les matematiques. Al llarg de la jornada escolar hi ha força moments per provocar situacions a resoldre: calcular les cartolines que ens faran falta per fer un mural, calcular la quantitat d'ametlla ratllada al moment de fer panellets, resoldre amb formes geometriques l'elaboració de mascares, preparar un berenar a la classe, fer grups per a la gimcana... Els problemes de magnituds i mesura, els de geometria i els de calcul... Totes aquestes situacions necessiten una planificacio, unes dades inicials, un objectiu final i saber organitzar el procés a seguir.

Davant d'un problema cal ajudar l'alumne/a a prendre consciència que hi ha alguna cosa a resoldre. Auquesta presa de consciencia serveix per voler comprendre la situacio i esbrinar d'on es parteix, que s'ha de resoldre i que ens falta per poder-ho fer i quines eines i estratégies es poden emprar. El mestre/a ha de facilitarque l'alum ne/a verbalitzi el que ha entes o be que ho representi usant dibuixos, esquemes, quadres de doble entrada..., i que ho comparteixi amb d'altres. Moltes vegades el fet d'explicar-ho als companys fa que s'ordenin les idees i es faciliti la comprensio.

Cal apropar els alumnes a diferents estrategies de resolucio, per tal d'afavorir l'aprenentatge de camins alternatius que els permetin véncer les dificultats $i$ la por davant d'un problema. En aquest sentit cal que el mestre/a utilitzi explícitament algunes estrategies didactiques com ara ajudar a llegir, analitzar, fer previsions de resultats, prendre consciencia de les dades conegudes i de les desconegudes, reformular que cal esbrinar, encadenar els diferents passos de raonament, realitzar les operacions numeriques, en el cas que es tracti d' un problema aritmetic, o els canvis d' unitats en d'altres i verbalitzar el procés seguit. Tambe pot proposar problemes de raonament logic que obliguen a llegir be la situacio, a transcriure-la, codificar-la, a fer quadres de doble entrada, a conjecturar i a preveure possibles solucions.

Es important oferir diferents presentacions dels problemes: orals, escrits, en imatges, amb TIC, etc. I en dife. rents contextos: reals, a partir d'un conte, matematicsi en d'altres àrees (coneixement del medi, música, plastica, etc.).

El mestre/a ha de deixar temps de reflexió i ha de transmetre que el proces és tan important com la solucio. Tambe ha d'afavorir l'intercami entre les diferents maneres de resoldre per l'enriquiment que representa.

Cal que el mestre/a proposi problemes amb una intenció estratégica concreta com el tempteig. l'analogia, la codificació, la representacio..., i en acabar es convenient que faci adonar als alumnes de quina han usat. En el cas de posar en comú la resolució de recerques o problemes oberts, la reflexio posterior hauria de portar a la presa de consciencia del procés de resolució dut a terme. D'aquesta manera es facilitara la transferència d'estrategies de resolucio.

Fuente: Departamento D'ENSENYAMENT, 2013a: 8. 
Como parte necesaria de las orientaciones metodológicas está la evaluación. Y ésta ha merecido un apartado específico en cada competencia. Las orientaciones para la evaluación tienen un carácter general para la competencia en cuestión, pero se llegan a concretar con la propuesta de unos cuantos indicadores, de acuerdo con los tres niveles de la gradación, que facilitan tal evaluación. Dado que las competencias determinadas deben servir de base para las evaluaciones externas que realice el Departamento de Enseñanza, se presenta también un ejemplo de prueba suficientemente amplia, que permita determinar el grado de dominio que de la competencia tienen los alumnos según las respuestas dadas. En todo caso, el ejemplo de evaluación mostrado no puede substituir nunca la necesidad de una evaluación continua que los docentes deben aplicar en las aulas, para constatar el avance que cada alumno hace en el camino del aprendizaje competencial.

En el momento de redactar este artículo, el Departamento de Enseñanza de la Generalitat de Cataluña había hecho pública la relación de competencias básicas curriculares siguientes:

- Competencias básicas del ámbito lingüístico de primaria y Eso (lenguas oficiales)

- Competencias básicas del ámbito matemático de primaria y EsO

- Competencias básicas del ámbito digital de primaria y ESO

- Competencias básicas del ámbito científico-tecnológico de la ESO

Y se trabajaba en los restantes ámbitos curriculares hasta llegar a cubrir todas las asignaturas del currículum, tanto de primaria como de la secundaria obligatoria. Paralelamente, se lleva a cabo una tarea de información y formación de los docentes para que los elementos novedosos que significan las competencias básicas se materialicen en la práctica docente cotidiana. A tal efecto se editan documentos específicos que pueden ser consultados en la web oficial del Departamento de Enseñanza (http://www.xtec.cat/web/currículum/competenciesbasiques). 


\section{CUADRO 6. EJEMPLO DE ORIENTACIONES PARA LA EVALUACIÓN DE UNA COMPETENCIA DEL ÁMBITO DIGITAL DE NIVEL PRIMARIO}

\begin{tabular}{|c|c|c|}
\hline Nivell 1 & Nivell 2 & Nivell 3 \\
\hline $\begin{array}{l}\text { Aplica funcions bàsiques per donar } \\
\text { format a documents de text. }\end{array}$ & $\begin{array}{l}\text { Aplica, amb pautes, diferents } \\
\text { formats a documents de text i hi } \\
\text { incorpora elements multimèdia, } \\
\text { enllaços, etc. }\end{array}$ & $\begin{array}{l}\text { Aplica diferents formats a } \\
\text { documents de text, i hi incorpora } \\
\text { elements multimèdia, enllaços, etc. }\end{array}$ \\
\hline $\begin{array}{l}\text { Utilitza les funcions bàsiques d'un } \\
\text { full de càlcul. }\end{array}$ & $\begin{array}{l}\text { Utilitza, amb l'ajut d'un tutorial, } \\
\text { diferents funcions d'un full de } \\
\text { càlcul, com ara fórmules } \\
\text { matemàtiques, que es requereixin } \\
\text { en l'activitat. }\end{array}$ & $\begin{array}{l}\text { Utilitza diferents funcions d'un full } \\
\text { de càlcul, com ara fórmules } \\
\text { matemàtiques, que es requereixin } \\
\text { en l'activitat. }\end{array}$ \\
\hline $\begin{array}{l}\text { Aplica funcions bàsiques per } \\
\text { elaborar presentacions. } \\
\text {... }\end{array}$ & $\begin{array}{l}\text { Seguint guies enriqueix les } \\
\text { presentacions amb funcions com } \\
\text { ara efectes, transicions, inserció } \\
\text { d'elements multimèdia, etc. } \\
\ldots\end{array}$ & $\begin{array}{l}\text { Incorpora i enriqueix les } \\
\text { presentacions amb funcions com } \\
\text { ara efectes, transicions, inserció } \\
\text { d'elements multimèdia, etc. } \\
\text {... }\end{array}$ \\
\hline
\end{tabular}

Tot seguit es mostra un exemple que permet avaluar el nivell d'adquisició:

A l'escola hi ha instal tlada una petita estació meteorològica. Es tracta d'un espai on, amb diferents aparells, termòmetre i pluviòmetre, s'enregistren dades atmosfèriques de forma continuada.

Durant el curs s'encarrega a l'alumnat de 6è que faci un recull de les dades obtingudes amb aquests instruments perquè elabori un informe que serà penjat al blog de l'aula.

Els encarregats d'observar i enregistrar les dades són els alumnes, que es distribuiran la tasca per torns: un d'ells cada setmana.

Es demana a l'alumne encarregat:

Has d'incorporar en el blog de l'aula el trets més significatius pel que fa a les dades meteorològiques de la setmana. Per fer-ho:

- Durant el període que tinguis assignat, anota, en un full de càlcul, les dades obtingudes amb l'estació meteorològica del centre. En concret has de recollir:

- la temperatura màxima i mínima,

- els litres d'aigua caiguts.

- Fes un tractament de les dades obtingudes que permeti visualitzar ràpidament l'oscil đlació tèrmica i la pluviometria. Aquest tractament pot incorporar càlculs estadístics, representacions gràfiques, etc.

Els qui ho necessiteu podeu consultar els tutorials que teniu al vostre abast.

Fuente: DePartament D'ENSENyAment, 2013c, 14. 


\section{REFERENCIAS BIBLIOGRÁFICAS}

COnferència Nacional D’Educació (2002) Debat sobre el sistema educatiu català. Departament d'Ensenyament, Generalitat de Catalunya.

GRUPO DE TRABAjo B (2004) Competencias clave para un aprendizaje a lo largo de la vida. Un marco de referencia europeo. Comisión Europea.

DePARTAMENT D'ENSENYAMENT (2000) Identificació de les competències bàsiques en l'ensenyament obligatori. Generalitat de Catalunya.

Departament D'EnSENYAMEnT (2003a) Congrés de competències bàsiques. Generalitat de Catalunya, Edición digital.

DEPARTAMENT D'ENSENYAMENT (2013a) Competències bàsiques de l'àmbit matemàtic. Identificació i desplegament de l'educació secundària obligatòria. Generalitat de Catalunya.

Departament D'ENSENYAMENT (2013b) Competències bàsiques de l'àmbit matemàtic. Identificació i desplegament de l'educació primària. Generalitat de Catalunya.

DEPARTAMENT D'ENSENYAMENT (2013c) Competències bàsiques de l'àmbit digital. Identificació $i$ desplegament de l'educació primària. Generalitat de Catalunya.

DEPARTAMENT D'ENSENYAMENT (2014) Competències bàsiques de l'àmbit cientificotecnològic. Identificació i desplegament de l'educació secundària obligatòria. Generalitat de Catalunya.

Mastache, A. (2007) Formar personas competentes. Buenos Aires, Noveduc.

PROYECTO "TunING" (2004) Afinar las estructuras educativas de Europa. http://tuning.unideusto.org/tuningeu/.

Rychen, D. S. y SAlganik, L. H. (eds.) (2003) Key competencies for a successful life and a well-functioning society. Publicaciones OCDE.

SARRAmona, J. (2000) Competencias básicas al término de la escolaridad obligatoria. Revista de Educación, 322, 255-290. 